Revue des patrimoines

25 | 2014

Le patrimoine de la Grande Guerre

\title{
Landowski, un sculpteur emblématique
}

\section{Michèle Lefrançois}

\section{(2) OpenEdition}

\section{Journals}

Electronic version

URL: http://journals.openedition.org/insitu/11580

DOI: $10.4000 /$ insitu. 11580

ISSN: 1630-7305

\section{Publisher}

Ministère de la Culture

\section{Electronic reference}

Michèle Lefrançois, "Landowski, un sculpteur emblématique », In Situ [Online], 25 | 2014, Online since 15 December 2014, connection on 29 June 2020. URL : http://journals.openedition.org/insitu/11580 ; DOI : https://doi.org/10.4000/insitu. 11580

This text was automatically generated on 29 June 2020.

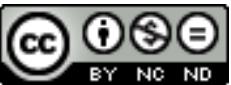

In Situ Revues des patrimoines est mis à disposition selon les termes de la licence Creative Commons Attribution - Pas d'Utilisation Commerciale - Pas de Modification 4.0 International. 


\title{
Landowski, un sculpteur emblématique
}

\author{
Michèle Lefrançois
}

\section{Un artiste dans la guerre}

1 Le 30 septembre 1915, Paul Landowski est intégré à la section camouflage d'Amiens, dirigée par le peintre Guirand de Scévola. Rattachée au $13^{\mathrm{e}}$ régiment d'infanterie, cette section rassemble de nombreux artistes (Jean-Louis Forain, Jacques Villon, Charles Despiau, Paul Albert Laurens, André Dunoyer de Segonzac, Maurice Denis, Édouard Vuillard...) chargés d'inventer des moyens de tromper l'ennemi en maquillant le matériel et les lieux. Landowski couvre alors ses carnets de croquis purement militaires, ou « croque » sur le vif la vie quotidienne dans les camps. Parallèlement, il tient un journal relatant les faits militaires. Dès qu'arrivent les premiers projets de monuments commémoratifs, le sculpteur se souvient des scènes qu'il a vues, des sentiments qu'il a éprouvés et se promet de « relever » ces morts. Il réalisera ce souhait avec quelque 25 monuments dédiés à la Première Guerre mondiale, érigés entre 1920 et 1935 sur le sol d'outre-mer (à Alger et à Casablanca), en Suisse et en Argentine. Landowski apparaît alors comme un sculpteur emblématique de ce type de statuaire.

\section{L'image de la guerre}

2 Grand Prix de Rome de sculpture en 1900, Landowski prit largement part à la commémoration funéraire de la Première Guerre mondiale avec d'ambitieux programmes iconographiques répondant à sa culture humaniste et à sa conviction sincère de la nécessité d'un hommage collectif aux victimes.

Comme pour tout monument de ce genre, le sculpteur n'échappa point à l'iconographie banalisée. Le même poilu dressa sa silhouette à Grand-Couronne (1920), au Neubourg (1920), à Combs-la-ville (1921), à Labarthe-Inard (1922), à Varengeville (1922), à Barcelonnette (1922), à Chézy sur Marne (1923). Plusieurs thèmes furent abordés. La vie 
dans les tranchées fut traitée en bas-reliefs à Bordeaux (faculté de médecine, 1921), à Paris (Hôtel-Dieu, 1921), à Saint-Quentin ${ }^{1}$ (1927). La figure féminine apaisante, symbole d'une Victoire reconnaissante déposant des couronnes, fut utilisée à Bagneux (1922) (fig. 1), à Annonay (1922), à Saint-Paul sur Ubaye (1922), à Buenos Aires ${ }^{2}$ (1923). Elle prit l'apparence d'une infirmière suisse à Schaffhouse (1922) ; celle de veuve et de mère à Boulogne-Billancourt (1923), à Fargniers (1926). Landowski en explique l'origine iconographique dans son Journal (16 février 1922) « [...] Je ferai cette statue à laquelle je pense depuis les premiers jours de la guerre : cette femme que j'ai vue un soir, adossée à un pilier de la gare d'Orsay et qui pleurait, les bras tombant, sans un geste ». Il ajoute : «[...] Le motif central est la douleur de la femme et de l'enfant» (Journal 7 octobre 1922).

Figure 1

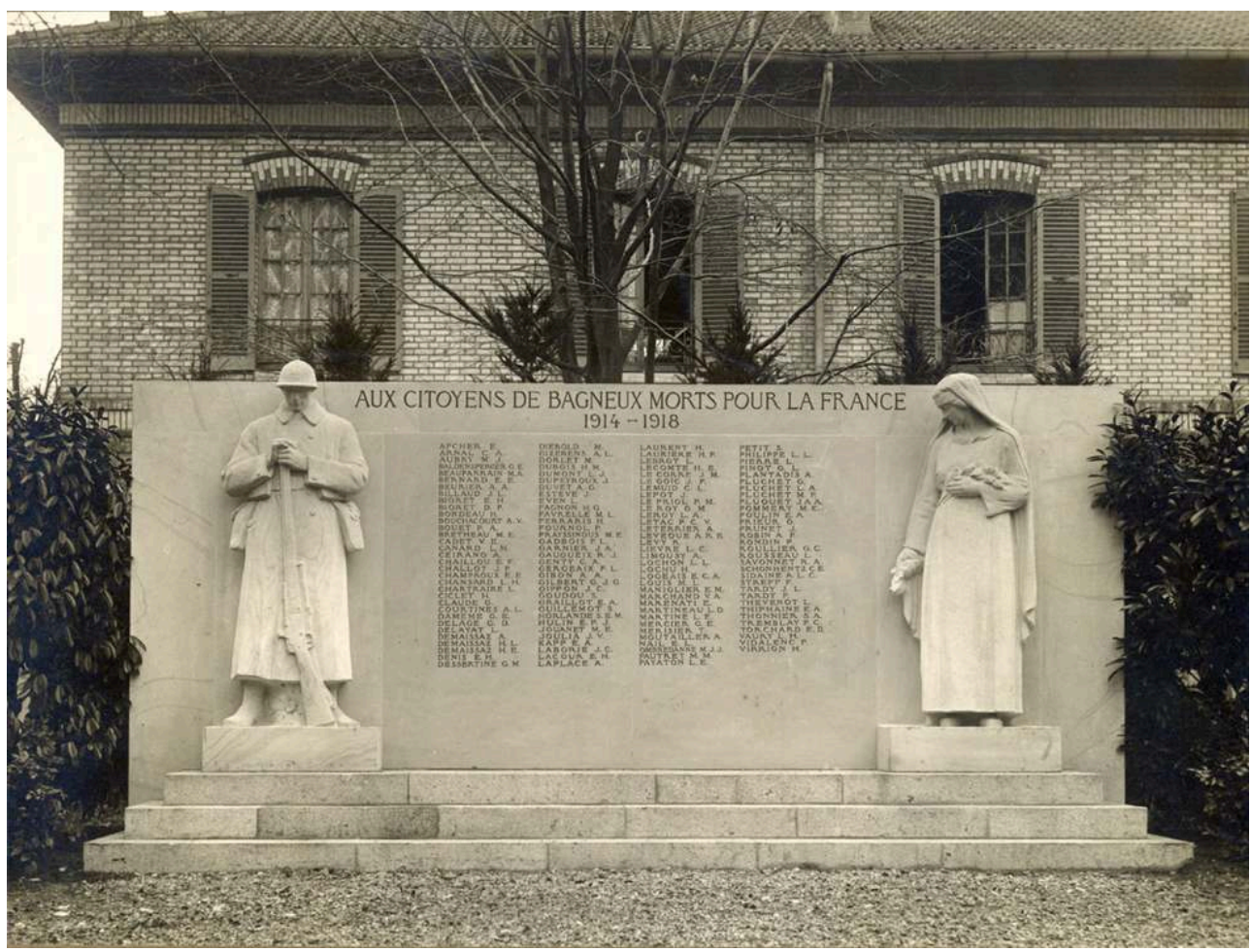

Bagneux (Hauts-de-Seine), square de la mairie : Monument aux morts, 1922. Pierre de Lens.

(c) Musées de la Ville de Boulogne-Billancourt. Fonds Landowski. Droits réservés.

Landowski s'accommoda de cette imagerie obligée, fidèle à la tradition figurative didactique qui permettait de façon directement explicite d'évoquer l'événement contemporain. Chez le sculpteur, l'allégorie fut rare. Cependant il y eut parfois recours comme sur le mur de fond du monument aux morts de l'École normale supérieure de Paris, où à côté de scènes militaires, apparaissent des sujets allégoriques sur la paix et le travail (fig. 2). Il note dans son Journal (12 mai 1919) : « [...] une suite de bas-reliefs évoquant la vie de la tranchée ». Un grand bas-relief couronnera le tout qui tâchera de donner une conclusion, comme une raison d'être à tant de sacrifices. Il renchérit : « [...] car il faut arriver pour ce monument, tout en évoquant les morts, évoquer le but de leur sacrifice. Le thème doit être évidemment la paix » (Journal 4 février 1920). 
Figure 2

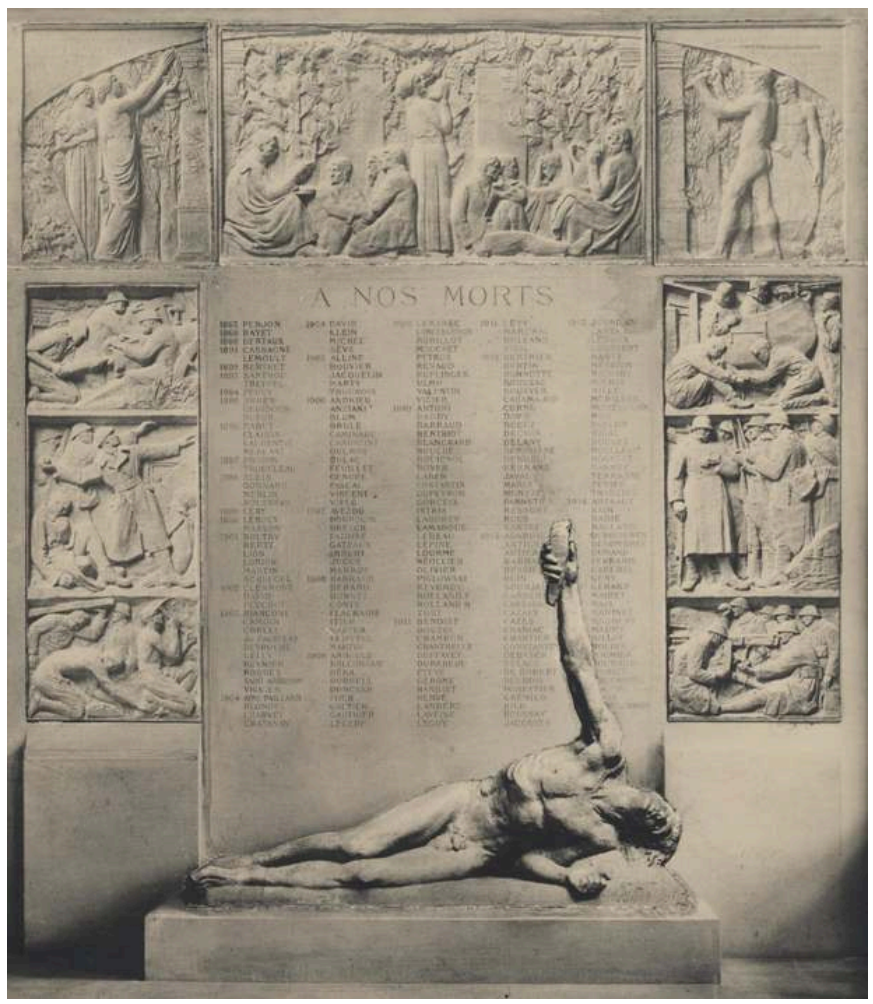

Paris, vestibule de l'École normale supérieure : Monument aux morts de l'École normale supérieure, 1923. Pierre.

Phot. Rosenman. (c) Musées de la Ville de Boulogne-Billancourt. Fonds Landowski.

5 En quelques occasions, Landowski déploya son sens de l'épique. Dans les jardins de la mairie parisienne du $16^{\mathrm{e}}$ arrondissement, une imagerie héroïque court sur un Bouclier (1928), tandis qu'en son centre caracole la figure ailée de l'épopée. La forme symbolique sert de cadre à l'évocation des batailles que livra la France pour sa libération. On y voit tour à tour sainte Geneviève, Jeanne d'Arc, les champs catalauniques, Jemmapes, Valmy, le retour de Metz et Strasbourg à la France... Ce bouclier est l'aboutissement de plusieurs projets remontant à 1920, qui devaient commémorer les fêtes de la Victoire et le cinquantenaire de la République.

7 Le plus célèbre monument, celui des Fantômes, érigé dans la plaine de Chalmont dans l'Aisne, mérite une attention particulière (fig. 3). 
Figure 3

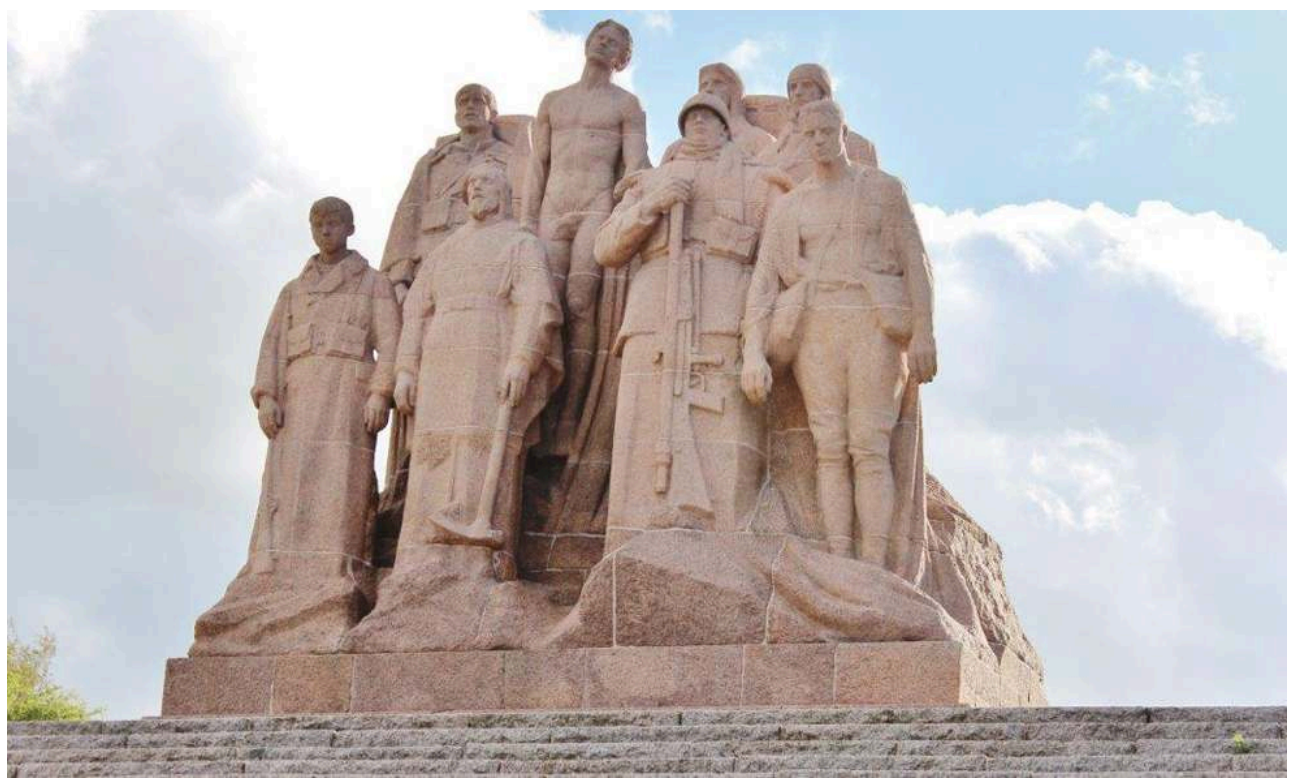

Aisne, plaine de Chalmont : Les fantômes, 1935. Granit.

Phot. Lefrançois, Michèle. (c) Michèle Lefrançois.

éphémères comme celles prévues à l'Arc de Triomphe. Landowski s'inquiète : «Pauvres poilus! Que va-t-on faire de vous!» s'interroge-t-il dans son Journal (5 juin 1918). Il imagine déjà son monument aux Poilus avec au centre, un tombeau où seraient enfermés les ossements d'un soldat inconnu ; autour, un cloître formé de quatre murs sculptés représentant l'infanterie, la marine, l'aviation. Le quatrième mur serait réservé aux mères et aux veuves. Au milieu des deux portes se dresserait la Victoire crucifiée. Extérieurement, une frise montrerait les armées en marche. Aux angles de soubassement, quatre groupes de héros évoqueraient les étapes de la libération de la France, incarnés par Sainte-Geneviève, Charles Martel, Jeanne d'Arc, Lazare Carnot. Des statues équestres seraient dédiées aux grands militaires.

Puis le projet de monument s'officialisant, le sculpteur propose une colonnade qui supporte une longue frise faisant tout l'hémicycle à gauche du rond-point des ChampsÉlysées en allant vers la Concorde. Au centre du groupe, les morts se relèvent pour voir passer la Victoire. Pour la première fois l'image des Fantômes qui se redressent prend corps. Mais des délais trop courts contraignent Landowski à refuser sa participation au monument éphémère de l'Arc de Triomphe qui sera finalement confié à Louis Sue, André Mare, Antoine Sartorio, et Gustave Jeaulmes. En revanche, l'État lui promet que son projet sera réalisé en dur, mais dans le futur. 

devra avoir le monument définitif. Pensons que l'événement que nous devrons commémorer, nous n'en soupçonnons pas encore les conséquences et son immense fécondité » (Journal 25 novembre 1918). Le ton est donné.

13 débattre. Deux projets se définissent peu à peu dans l'imagination du sculpteur. Le Mort porté par le peuple (gisant porté par des hommes et des femmes) et à nouveau Les Fantômes (groupe de soldats se relevant de terre). Leur iconographie mérite d'être comparée. Le Mort porté par le peuple relève de la plus pure tradition commémorative. Landowski hésite entre les deux projets et imagine le lieu idéal: "La Troisième République se doit à elle-même d'élever à son tour son monument qui serait comme le Parthénon de la France. L'emplacement de ce monument, on en parla voici longtemps, l'idée aurait plu, elle était excellente, c'était le mont Valérien. Quel symbole ce serait d'abord d'élever là, à la place de ce fort, sur cette admirable terrasse, avec cette vue extraordinaire que l'on a de là sur Paris, d'élever là en plein ciel, le monument du héros inconnu, monument à la fois à tous nos morts, monument à la paix, monument à la France... Mais je sais les grosses difficultés... C'est alors sur les Invalides qu'il faudrait se rabattre. L'endroit est recueilli. Je vois très bien la transformation de l'esplanade des Invalides en une voie triomphale... Je vois très bien le tombeau du héros dans le square de gauche... l'autre square consacré à la gloire des autres héros anonymes de la guerre » (Journal 10 novembre 1920). Le lieu des Invalides semblent l'emporter et Landowski dessine in situ les deux iconographies et les commente: "[...] présenter mes deux groupes, soit Les Fantômes soit le Mort porté par le peuple. L'idée de défoncer le square pour faire cette sorte de cloître à ciel ouvert est très bonne. Surtout pour le monument porté... Pour Les Fantômes, ils [doivent] sembler réellement surgir du sol ».

14 d'un monument appelé Les Morts. Le titre utilisé par Landowski est banal, bien qu'il ait déjà choisi l'image des Fantômes, préférée à celle du gisant. Mais, explique-t-il, « pour la commande, il ne faut effrayer personne » (Journal 17 octobre 1919). La sculpture obtient la médaille d'honneur au Salon des Artistes français en 1923. Dès lors, son lieu d'érection sera successivement envisagé à Verdun, à l'ossuaire de Douaumont pour finalement être fixé près d'Oulchy-le Château, sur la butte de Chalmont qui vit la seconde bataille de la Marne, essentiellement la journée du 28 juillet 1918, durant laquelle les troupes allemandes durent se replier vers la Vesle. La commande officielle d'un monument en granit intervient en juillet 1926.

Sur la butte de Chalmont, le groupe sculpté apparaît de loin comme un roc solitaire dressé en terrain plat. Des silhouettes humaines semblent se relever du sol. Ce sont sept soldats. L'artilleur, le grenadier, le mitrailleur, l'aviateur, le sapeur, le territorial, le petit paysan. Ils ont les paupières closes. Au milieu d'eux se dresse un jeune homme nu, figure du héros mort. Plus bas, devant, se dresse la statue de la France. L'ensemble est grandiose et inspire le recueillement. Idée de la mort et de la résurrection. La jeunesse s'est sacrifiée mais les morts revivent par la mémoire collective. Les soldats, devenus fantômes, se libèrent du champ meurtrier des batailles, entraînés par la silhouette dressée du jeune martyr: «La figure la plus difficile, à cause de son caractère symbolique, écrit Landowski, ce n'est pas un modèle athlétique, mais un adolescent plutôt frêle à très beau visage. La faiblesse, la jeunesse, la beauté » (Journal 
14 janvier 1921). Une statue de la France les précède. Quatre paliers les séparent, symbolisant les quatre années de combats.

Figure 4

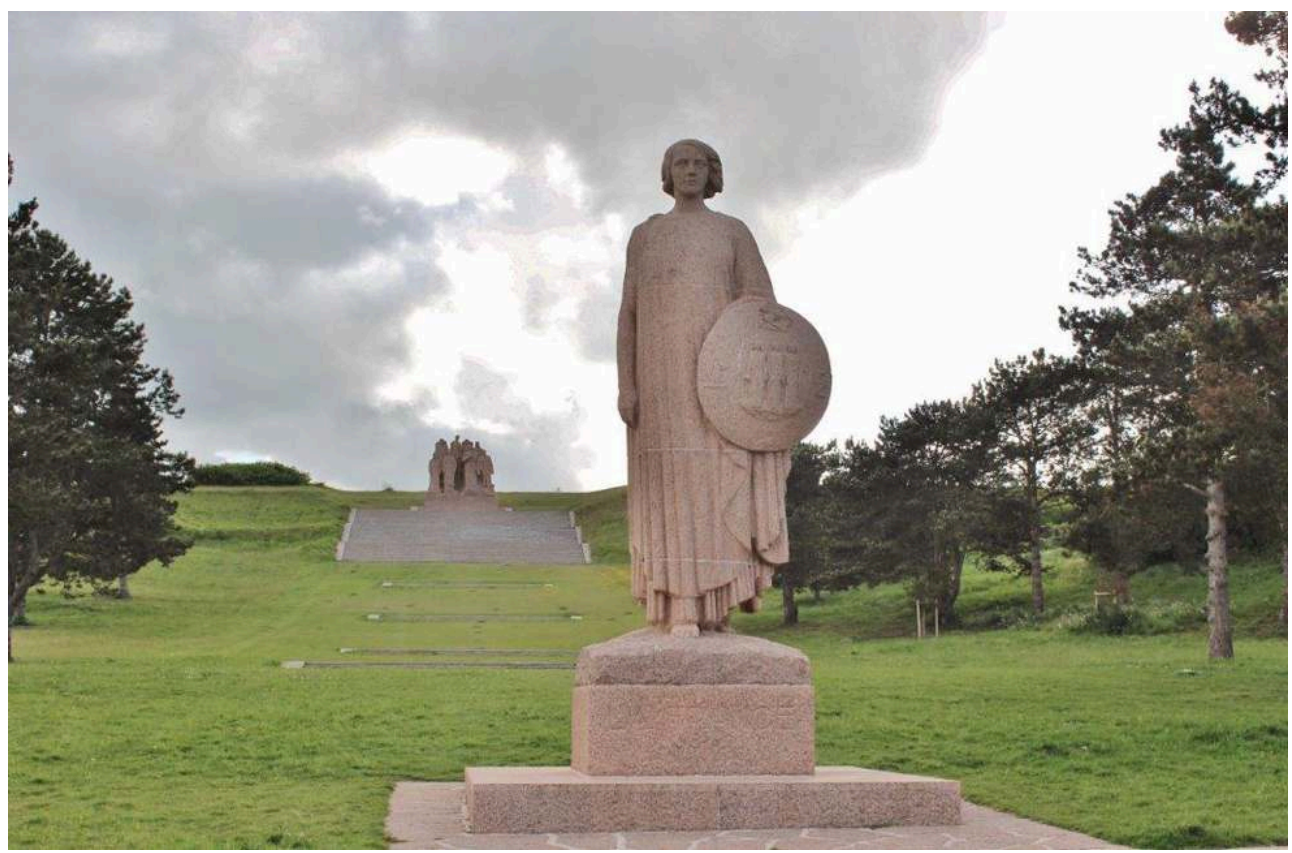

Aisne, plaine de Chalmont : La France, 1935, Granit.

Phot. Lefrançois, Michèle. (c) Michèle Lefrançois.

C'est en 1928, après le choix de l'emplacement, que Landowski a «trouvé la définitive présentation des Fantômes [...]. Défoncer la colline, d'où jailliraient les morts dressés [...] Des paliers autant que les années de guerre, et au bord de la route, une grande figure de la France en marche. Le paysage et la sculpture intimement liés» (Journal 29 novembre 1928). Quant à $L a$ France, elle aura fait l'objet de nombreuses réflexions et études (fig. 4). Pendant une année, de 1930 à 1931, Landowski multiplie les projets. Il note : «Cette fois, je crois vraiment tenir la France 1918 pour Chalmont. Elle avance toute droite sans un geste. Un vent léger dans les cheveux, et le grand manteau agrafé sur l'épaule droite. La seule arme qu'elle porte est défensive. C'est le bouclier portant les trois déesses qui font la devise de la France : Liberté, Égalité, Fraternité... » (Journal $1^{\text {er }}$ décembre 1930). Mais avant ce choix, que d'hésitations, de repentirs, dont le Journal du sculpteur rend compte. Il existe, d'après les archives conservées, neuf versions de la statue. Sur l'une, la France porte un bonnet phrygien et tient un glaive (fig. 5). Mais d'emblée, Landowski écarte l'idée d'une France guerrière et explique que le glaive symbolise ici la justice. Se pose également la question du vêtement. Sera-t-il antique ou moderne? Le plus spectaculaire imaginé un temps par le sculpteur est une sorte de chasuble portant des bas-reliefs historiés de l'histoire nationale, « un ample manteau, comme celui des rois de France, tout brodé de bas-reliefs, criblé de figures. Histoire de la France. Les légendes historiques. Tout cela devrait être exécuté en matières précieuses. Ivoire, or, argent $^{3}$ ». Le bonnet phrygien, qui un court moment couvre sa tête, disparaît pour libérer la chevelure qui ondule dans le vent. Manifestement, Landowski mise sur la simplicité. Il veut une France proche : «Je ne veux pas que ce soit encore une Minerve. Je ne veux pas que ce soit une ressuscitée de la Victoire de Rude, 
ni une Samothrace. Il faut qu'elle soit énergique et douce. Il faut qu'elle ait de l'action. Il ne faut pas qu'elle gesticule. Il faut que ce soit une force irrésistible en marche » (Journal 20 mai 1931). Le bouclier que porte la France, lui aussi, évolue. D'une iconographie historique qui retrace les grandes batailles nationales, il passe à la simple évocation des trois figures républicaines. Car l'histoire de ce bouclier est ancienne. Elle commence en 1920, lors d'un concours lancé pour la commémoration des fêtes de la Victoire et du cinquantenaire de la Troisième République. Le Bouclier est destiné au Panthéon ou à l'Arc de Triomphe. Landowski conçoit alors plusieurs projets. Le texte gravé retrace les grandes étapes de l'histoire nationale ${ }^{4}$. Les bas-reliefs, très fins, sont répartis en zones concentriques. Son image fait la page de couverture de L'Illustration du 20 novembre 1920. Puis la forme successivement ovale, triangulaire, ronde, ainsi que le motif central, changent. À un motif de Victoire cavalière, Landowski préfère les trois figures républicaines qu'il reprendra pour la France des Fantômes.

\section{Figure 5}

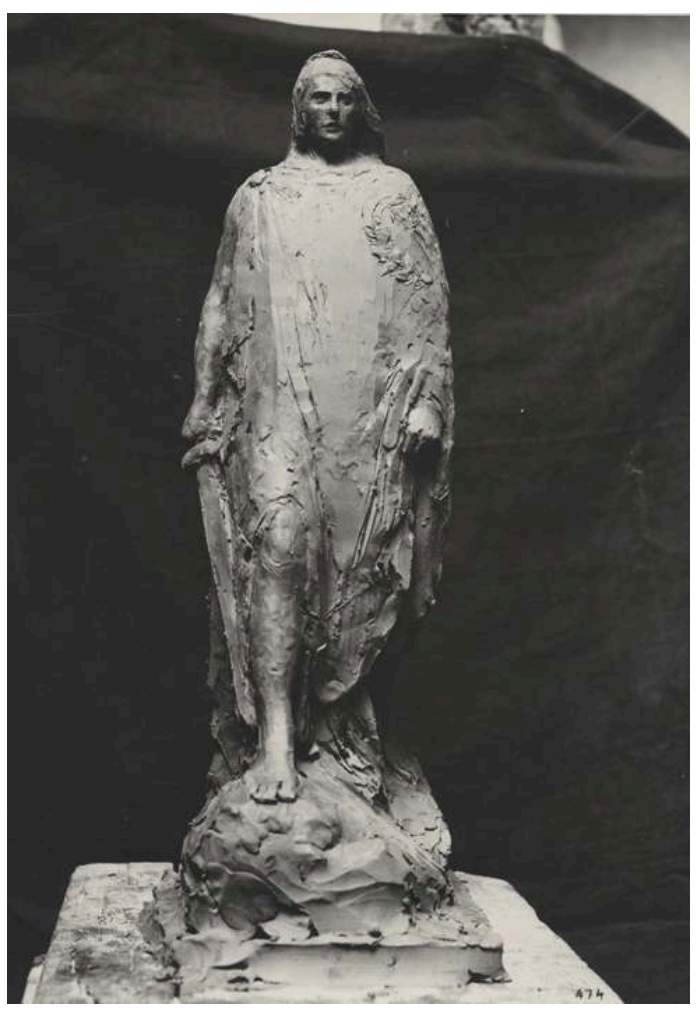

Boulogne-Billancourt (Hauts-de-Seine), Musée des années 30 : La France (étude), 1930, Plâtre. (c) Musées de la Ville de Boulogne-Billancourt. Fonds Landowski. Droits réservés.

17 Avec Les Fantômes, dont il regrettait de ne pas les avoir faits plus grands car « il aurait fallu cinq ou six mètres de plus!» (Journal 22 juin 1934), Landowski s'affirmait comme un maître de la sculpture monumentale commémorative.

Landowski était de la tradition des modeleurs. Mais il trouva cependant « une joie physique à travailler la pierre » (Journal 22 juillet 1930). Il multiplia les études en granit pour tester la matière, et monta quotidiennement sur l'échafaudage.

19 Le monument est inauguré avec solennité le 27 juillet 1935, par le président Albert Lebrun, soit une quinzaine d'années après les premières esquisses. 

combattants de la Première Guerre mondiale, La Victoire 6 . Le monument est inauguré par le maréchal Lyautey et le Pacha de Casablanca en 1924 (fig. 6). Il représente deux hommes à cheval, un spahi marocain et un cavalier métropolitain, se faisant face et se serrant la main. Landowski se souvient: "L'idée me vient soudain de réaliser là une scène que j'ai vue dans la campagne romaine : deux gardiens de troupeaux à cheval, se rencontrant, vinrent l'un vers l'autre, et se donnèrent la main par dessus l'encolure de leur monture. Geste symbolique » (Journal 19 mai 1921). En 1961, l'association Le Burnous fit transporter le monument pour le réédifier à Senlis.

\section{Figure 6}

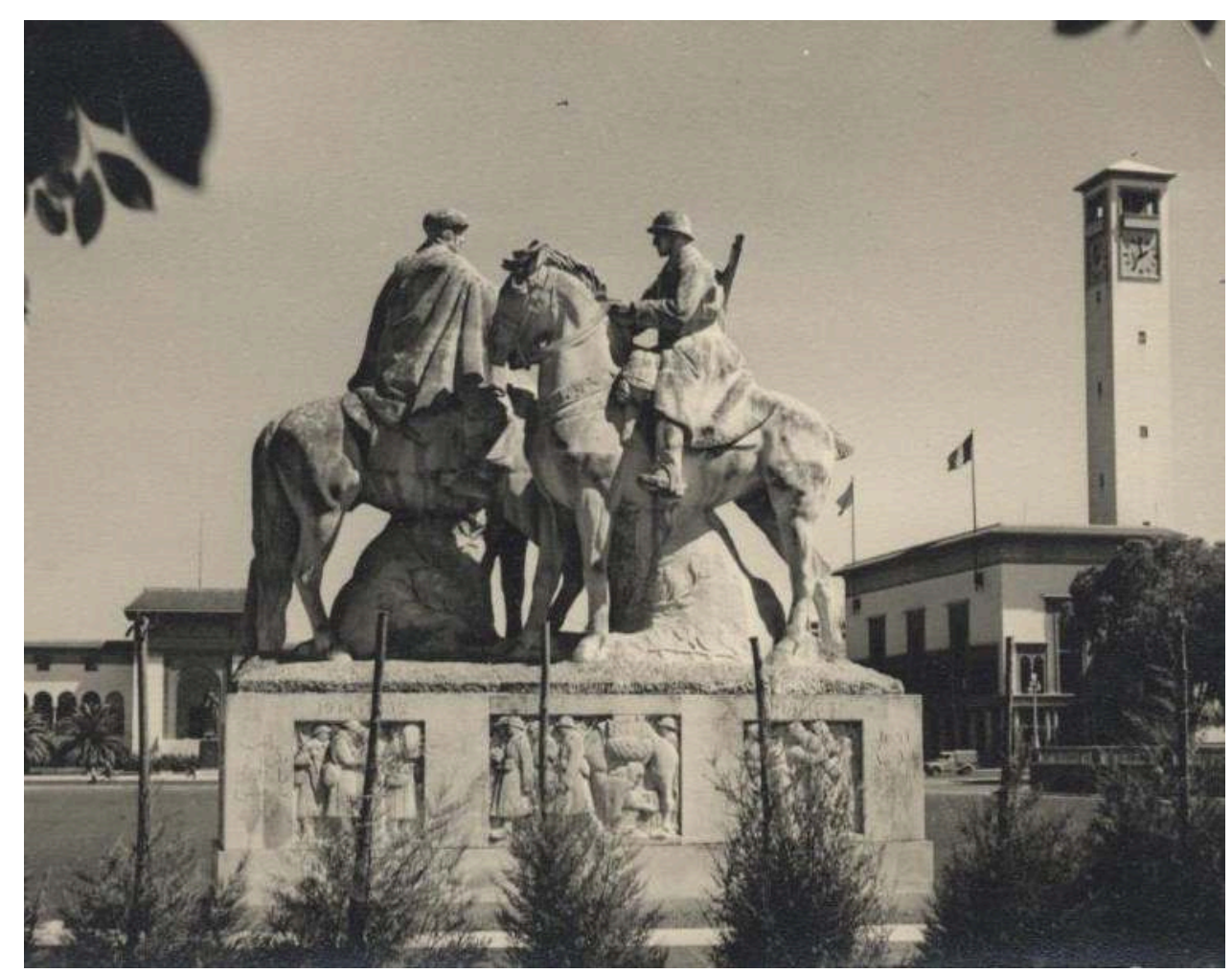

Casablanca (déplacé à Senlis, place du 3è Houzard) : La Victoire, 1924, Pierre de Lens. Phot. Durand, G. (c) Musées de la Ville de Boulogne-Billancourt. Fonds Landowski. 
Quatre ans plus tard, c'est à Alger que Landowski manifesta à nouveau son sens spectaculaire de l'intégration du monumental dans l'espace avec le monument aux morts intitulé Le Pavois (1928) (fig. 7). Situé au sommet d'une volée de marches, dominant la baie, la sculpture écrivait son auteur, devait avoir «un caractère somptueux et héroïque qui devait faire bien sous le soleil d'Afrique " (Journal 28 février 1921). L'image choisie est celle de deux cavaliers, métropolitain et algérien, entourant une victoire ailée, tous trois portant le gisant d'un soldat mort. À l'arrière du groupe central, un groupe évoque la vie communautaire ${ }^{7}$.

Figure 7

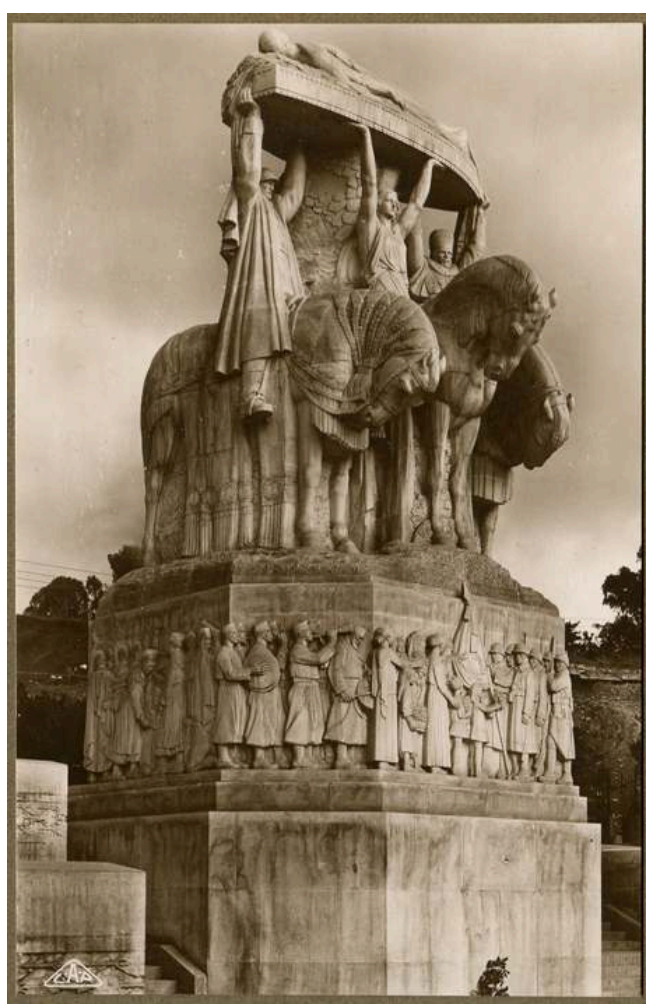

Alger, boulevard Khémisti : Le Pavois, 1928. Pierre de Lens.

Phot. «Réal photo ». (c) Musées de la Ville de Boulogne-Billancourt. Fonds Landowski.

Après l'indépendance de l'Algérie, le monument fut caché sous un coffrage de ciment.

Honoré pour l'un, exilé ou dissimulé pour les autres, ces trois derniers monuments de Landowski sont exemplaires de l'impact de la sculpture publique commémorative sur l'émotion commune. À des titres différents, tous trois témoignent de l'histoire du drame humain qui s'est joué durant la Grande Guerre. Gardiens visibles d'une mémoire collective, ils constituent un patrimoine artistique qui a prétention à défier l'oubli. 


\section{BIBLIOGRAPHY}

- Paul Landowski, Journal, inédit. Publié en ligne sur le site journal.paul-landowski.com

- Arch. nat. F21 4230

- LEFRANÇOIS, Michèle. « Landowski, l’œuvre sculpté ». Paris : Créaphis, 2009.

- LEFRANÇOIS, Michèle. «Les Fantômes ». Dans COMPÈRE-MOREL. Paul Landowski, la pierre d'éternité , cat. exp. Péronne, Historial de la Grande Guerre, mars-avril 2004. Paris : Somogy, 2004, p. 13.

- LEFRANÇOIS, Michèle. «Les Fantômes ». Dans BOUDOU, Dominique, LEFRANÇOIS, Michèle. Paul Landowski. Le Temple de l'Homme. Cat. Exp. Paris, musée du Petit Palais, décembre 1999-Mars 2000. Paris : Paris-Musées, 1999, p. 222.

- LEFRANÇOIS, Michèle. « Paul Landowski ». Dans RIVÉ, Philippe, BECKER, Annette, PELLETIER, Olivier. Monuments de mémoire. Monuments aux morts de la Grande Guerre. Secrétariat d'État aux anciens combattants et victimes de la guerre. Paris : Mission permanente aux commémorations et à l'information historique, 1991, p. 221.

\section{NOTES}

1. - Le monument fut réalisé en collaboration avec Henry Bouchard.

2. - Id.

3. - Inscriptions manuscrites sur dessin.

4. - PROCLAMATION DE LA REPUBLIQUE 1870/LE 14 JUILLET 1789/SAINTE GENEVIEVE/AN 1000 FONDATION DES COMMUNES/CHARLES MARTEL/1356 LES PREMIERS ETATS GENERAUX/ STRASBOURG 1944 PARIS/LA NUIT DU 4 AOUT/DUGUESCLIN/L'ARBRE DE LA LIBERTE/VALMY/ JEANNE D'ARC/LE DRAPEAU TRICOLORE/DECLARATION DES DROITS DE L'HOMME/MARNE 1918 VERDUN/

5. - Le paquebot désarmé, la statue fut acquise par un italien qui en fit don à la ville d'Altavilla Vicentina (Vénétie) en 2003.

6. - Place du Troisième Houzard.

7. - Les bas-reliefs du socle furent sculptés par Bigonet.

\section{ABSTRACTS}

Author of some twenty-five war memorials sculpted between 1920 and 1935, Landowski emerges as an artist whose work is emblematic of the First World War. The present study of this heritage is based on various direct sources such as Landowski's own journal and his correspondence. These sources help us understand his convictions as a man and as an artist, whilst the sketches and marginal annotations he made during the time he was incorporated into the "camouflage section' help understand his creative processes. The examination of the surviving archive comprising numerous models and sketches allows us to reach a better appreciation of the 
evolution of the iconography of his statues. Alongside other commemorative monuments, this present study pays particular attention to one of his major pieces, 'Les Fantômes', erected on the site of some of the war's bloodiest fighting during the second battle of the Marne. The article follows the ten years of hesitation that it took before this statue was completed. Special attention is also paid to Landowski's war memorials outside France, which have a particular historic charge.

Avec quelque 25 monuments aux morts sculptés entre 1920 et 1935, Landowski apparaît comme un artiste emblématique de la Grande Guerre. L'étude de ce patrimoine, témoin d'un immense drame humain, s'appuie sur des sources directes comme celle, essentielle, du Journal de Landowski, de sa correspondance qui aide à cerner ses convictions d'homme et d'artiste, de ses croquis réalisés durant son incorporation à la "section camouflage", de ses annotations en marge qui permettent de suivre le processus créateur. L'examen du fonds conservé de nombreuses maquettes et dessins permet également d'appréhender au plus près l'évolution de l'iconographie de la statuaire. En marge d'autres monuments commémoratifs, la recherche se focalise sur l'oeuvre majeure de Landowski dans ce domaine, Les Fantômes, qui se dressent à l'emplacement même des combats meurtriers. Elle retrace les dix années aléatoires nécessaires à leur réalisation. Une place particulière est réservée aux monuments d'outre-mer qui portent en eux une charge historique particulière.

\section{INDEX}

Mots-clés: Landowski, sculpteur, sculpture, monuments, commémoration, fantôme, journal, outre-mer, camouflage, iconographie

Keywords: Landowski, sculptor, sculpture, monuments, commemoration, ghost, journal, overseas, camouflage, iconography

\section{AUTHOR}

\section{MICHĖLE LEFRANÇOIS}

Docteur en histoire de l'art, Ancienne conservatrice des musées de Boulogne-Billancourt michele.lefrancois@free.fr 